in vivo $35: 871-881(2021)$

doi:10.21873/invivo.12327

\title{
Bone Morphogenetic Protein 7 Expression in Alveolar Bone Addition With Autologous Blood, Lyophilized Bone and Atelocollagen
}

\author{
BOGDAN-CĂTĂLIN ALEXANDRU ${ }^{1}$, MONICA POPA $^{1}$, LIVIU OANA ${ }^{2}$, COSMINA IOANA BONDOR $^{3}$, \\ CARMEN GEORGIU ${ }^{4}$, CAMELIA-SIDONIA LAZĂR ${ }^{4}$, ALINA SIMONA ȘOVREA ${ }^{5}$, \\ ANNE-MARIE CONSTANTIN ${ }^{5}$ and GABRIELA DOGARU 6
}

\author{
${ }^{1}$ Discipline of Medical Hygiene, "Iuliu Hațieganu” University of Medicine and Pharmacy, Cluj-Napoca, Romania; \\ ${ }^{2}$ Department of Surgical Pathology, University of Agricultural Sciences and Veterinary Medicine, Cluj-Napoca, Romania; \\ ${ }^{3}$ Department of Medical Informatics and Biostatistics, "Iuliu Hațieganu”" \\ University of Medicine and Pharmacy, Cluj-Napoca, Romania; \\ ${ }^{4}$ Discipline of Pathology, Department of Morphological Sciences, \\ "Iuliu Hațieganu” University of Medicine and Pharmacy, Cluj-Napoca, Romania; \\ ${ }^{5}$ Discipline of Histology, Department of Morphological Sciences, "Iuliu Hațieganu” \\ University of Medicine and Pharmacy, Cluj-Napoca, Romania; \\ ${ }^{6}$ Discipline of Medical Rehabilitation, "Iuliu Hațieganu” University of Medicine and Pharmacy, Cluj-Napoca, Romania
}

\begin{abstract}
Background/Aim: The biomaterials used in guided bone regeneration have undergone significant diversification in recent years. This study aimed to evaluate alveolar bone addition and bone morphogenetic protein 7 (BMP7) expression using an improved autologous and xenogeneic biomaterial. Materials and Methods: Chronic marginal periodontitis was induced in sheep; the intervention group received bone addition as periodontal therapy, using a composite system with lyophilized bovine bone enriched with atelocollagen type 1, platelet-rich plasma and advanced platelet-rich fibrin (A-PRF). Six weeks after the intervention, the dentoalveolar structures were evaluated using hematoxylin-eosin and immunohistochemical staining, to evaluate bone addition and BMP7 expression. Results: The untreated sheep showed inflammation, periodontal ligament destruction, remnants of calculus and bacterial plaque as well as foreign bodies in the desmodontal space, without
\end{abstract}

This article is freely accessible online.

Correspondence to: Carmen Georgiu, MD, Ph.D., Lecturer, Discipline of Pathology, Department of Morphological Sciences, "Iuliu Hațieganu" University of Medicine and Pharmacy, 8 Victor Babeș Str., 400012, Cluj-Napoca, Romania. Tel: +40 749284737 , e-mail: carmengeorgiu@hotmail.com

Key Words: BMP7, bovine xenograft, PRP, platelet-rich plasma, A-PRF, advanced platelet-rich fibrin. sings of repair. In the treated sheep, fibroblasts/fibrosis, cartilage and/or new bone, cellular cementum and desmodontium, along with remnants of biomaterial with various degrees of cellularity were observed. In the untreated group, the presence of BMP7 was found in osteoblasts and osteocytes while in the treated group, it was mainly found in the biomaterial remnants, while immunohistochemical staining was less intense in the newly formed osteoperiodontal tissues. Quantitative analysis using the MannWhitney U-test showed highly statistically significant differences between the two groups, demonstrating the efficiency of this composite system. Conclusion: The current composite system meets all the necessary conditions for promising guided alveolar bone regeneration.

Over the past years, special attention has been given to biomaterials used for guided alveolar bone regeneration (GTR); the best results in GTR have been shown by bone autografts and biomaterials containing recombinant growth factors. However, bovine xenografts have also been among the biomaterials of choice, and platelet-rich plasma (PRP) and platelet-rich fibrin (PRF) autologous blood derivatives have also been frequently mentioned as sources of growth factors. One of these growth factors, bone morphogenetic protein BMP-7, which belongs to the transforming growth factor beta (TGF- $\beta$ ) family and is involved in osteoblast differentiation with a main role in the transformation of mesenchymal stem cells into bone and cartilage, is predominantly found in autologous blood derivatives (1). In this regard, the 
a

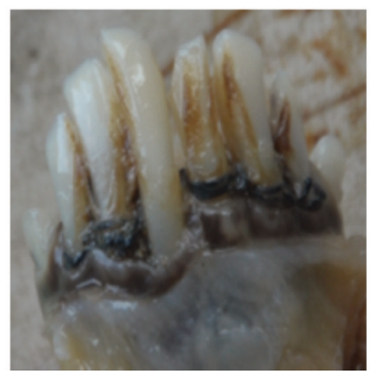

b

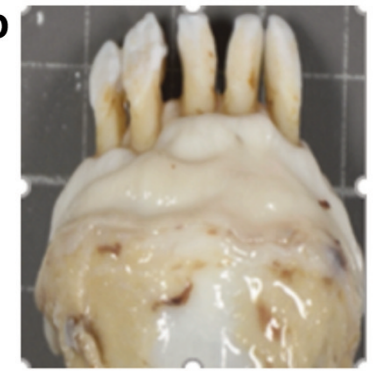

C

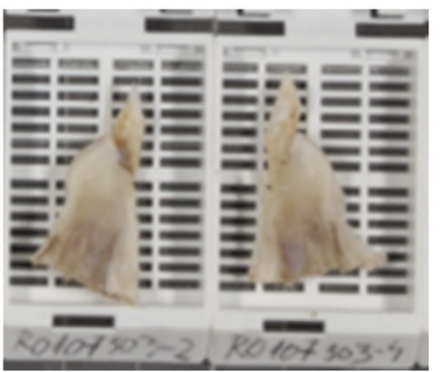

Figure 1. Macroscopic appearance of: (a) The untreated chronic marginal periodontitis; (b) A sample (48 hours after fixation in 10\% formalin), taken six weeks after bone addition; (c) Longitudinally sectioned samples prepared for paraffin embedding.

improvement of these biomaterials, especially by certain combinations, might have a beneficial effect on GTR. For this purpose, xenografts of bovine origin with a macroporous structure, enriched with atelocollagen type 1, responsible for osteoconduction, might be used. Atelocollagen type 1 stimulates and accelerates new bone formation, by initiating platelet aggregation and releasing growth peptides like TGF$\beta$, platelet-derived growth factor (PDGF), vascular endothelial growth factor (VEGF) and insulin growth factor (IGF) (2, 3). Regarding PRP, different studies have demonstrated its role in bone regeneration (4-12). In order to obtain PRP, test tubes with heparin addition can be used for the optimization of growth factor release (13). Besides its anticoagulant effect, heparin presents growth factor binding properties, allowing the biomimetic sequential release of these growth factors $(2,13)$. The advanced platelet-rich fibrin (A-PRF), unlike PRF, has an increased content of platelets, erythrocytes, neutrophils/granulocytes (leukocyte subfamilies) and an increased amount of stem cells (1). Moreover, the APRF membrane presents high amounts of growth factors, stimulating the cell proliferation and differentiation $(14,15)$. A-PRF can thus be used as an augmentation mass for hard tissues, both alone or in combination with other bone addition materials (1).

Until now, sheep have been used in many bone regeneration studies, including periodontal alveolar bone regeneration (16-19). The current experimental study was performed on sheep with chronic marginal periodontitis, a disease very similar in sheep to that found in humans. The aim of the current experimental study was to evaluate the alveolar bone regeneration on sheep, after a treatment using a combination of different biomaterials, a composite system containing bovine xenografts enriched with atelocollagen type 1, combined with PRP and covered with membranes made of A-PRF. Another aim of this study, was to evaluate the presence of bone morphogenetic protein 7 (BMP7) in the bone addition areas. All parameters were evaluated 6 weeks after the intervention.

\section{Materials and Methods}

\section{Animal groups}

Experimental group. The study was conducted based on the Ethics Committee's approval no 23 of 23.01.2015, issued by "Iuliu Hațieganu" University of Medicine and Pharmacy, Cluj-Napoca. The study included five sheep (Ovis aries, Romanian Turcana breed), aged five years, non-pregnant, multiparous females, with a weight ranging between $40-60 \mathrm{~kg}$, without any cardiac diseases or other organic disorders, but with chronic marginal periodontitis. Three of these sheep received periodontal treatments, including bone addition, representing the treated group. In order to avoid animal suffering, general anesthesia was used before any examination or intervention on the dento-periodontal structures (20, 21). After the diagnosis of chronic marginal periodontitis, prophylactic treatments and closed field periodontal curettage, followed by alveolar bone addition using a composite system containing Hypro-Oss preparation (Bioimplon GmbH, Gießen, Germany) were performed. The Hypro-Oss preparation consists of lyophilized original bovine bone enriched with atelocollagen type 1 , with granule sizes ranging between $0.5 \mathrm{~mm}$ and $1 \mathrm{~mm}$. PRP was obtained from autologous blood by centrifugation of glass test tubes containing heparin. The test tubes also contained a thixotropic gel that separated blood into the two fractions. The A-PRF membranes were obtained following centrifugation of autologous blood in simple vacuum test tubes. After centrifugation, the platelet clot was extracted and pressed, obtaining the A-PRF membranes. The membranes were cut and placed over the Hypro-Oss combined with PRP, covering the entire grafts, then placed on the vestibular part of the surgically treated periodontal structures. Subsequently, the mucoperiosteal flap was reapplied, also covering the biomaterial, and adequately sutured coronally, at cervical level. Antibiotic therapy was performed, using $1 \mathrm{ml}$ Amoxicillin Retard 20\% per 10 $\mathrm{kg}$ live body weight; the suspension was administered after stirring the vial in the neck muscle $(22,23)$, for a period of five days, starting 12 hours before initiation of the periodontal treatments.

Control group. In order to compare and quantify the results, the two other multiparous female sheep, left with untreated chronic marginal periodontitis were also studied, representing the control or untreated group (Figure 1A). All five animals were euthanized and dentoalveolar samples were collected after the same period of six weeks after the initial diagnosis, with or without treatment (Figure 1B). 
a

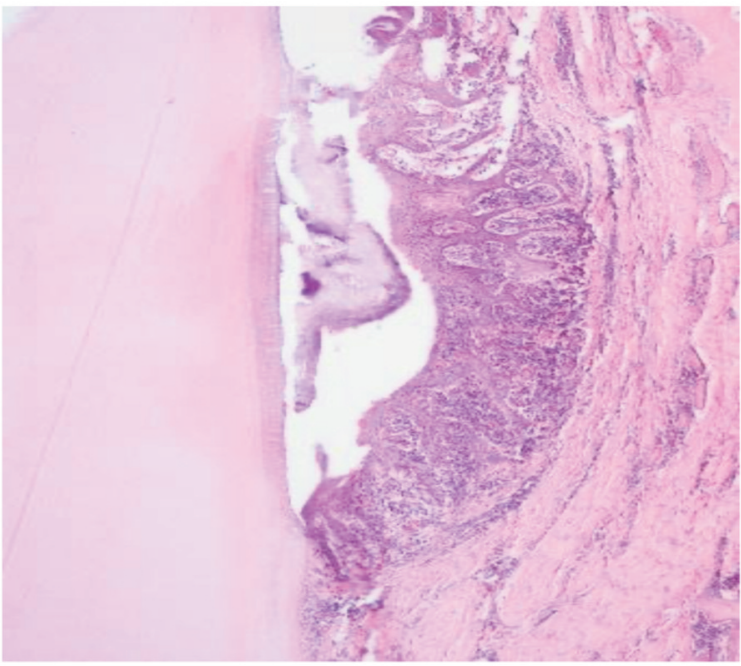

C

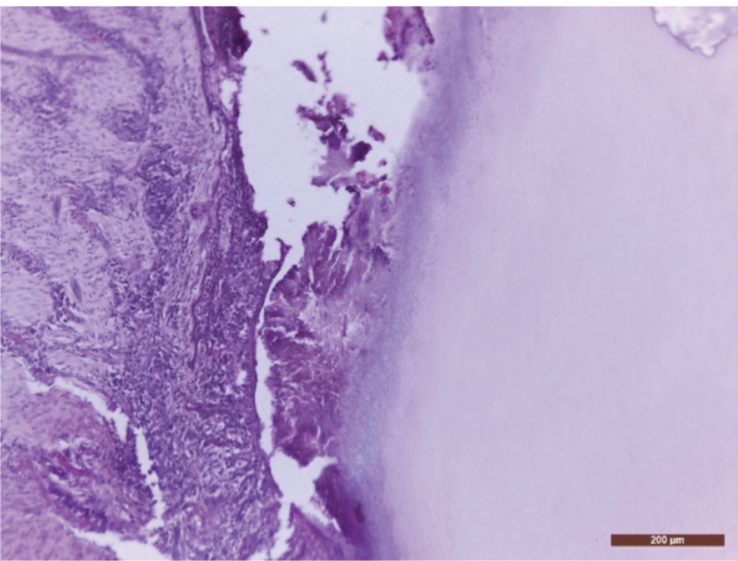

b

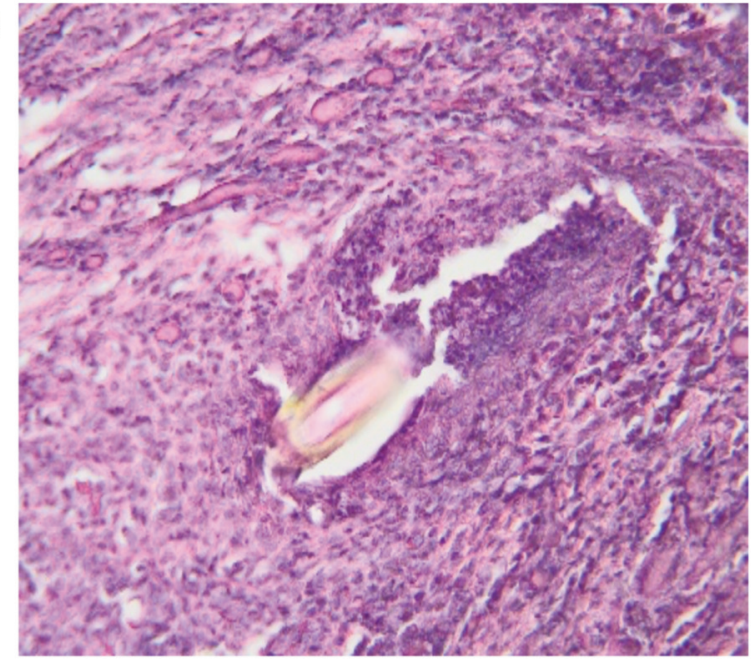

d

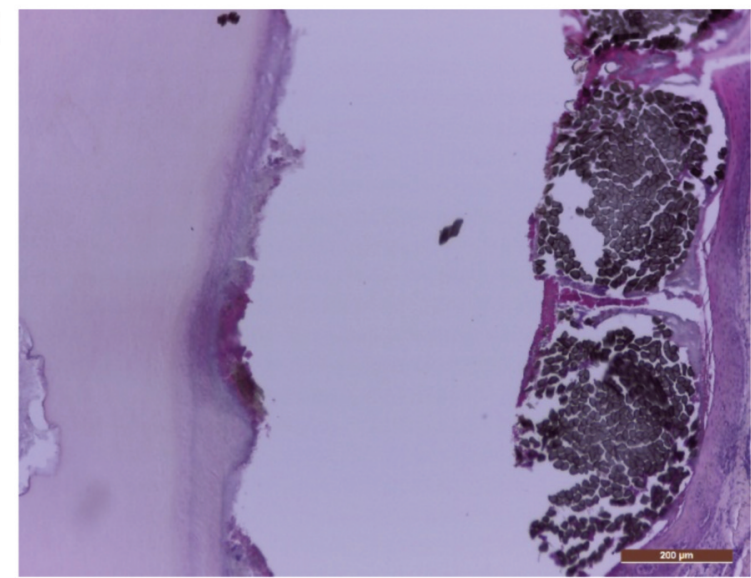

Figure 2. (a) Mineralized bacterial plaque on the root surface accompanied by inner epithelia of the marginal gingiva with chronic inflammation (100x); (b) Foreign body enclosed in the inner gingival epithelium (200x); (c) Calculus in the gingival sulcus, inflammation of the inner epithelium, destruction of the alveolar bone, as well as a dental carious process (100x); (d) Carious process, external dental resorption, foreign body in the gingival sulcus, inflammation of the inner epithelium (100x). Hematoxylin-eosin staining.

Staining techniques. In both animal groups, histological and immunohistochemical examinations were performed in order to evaluate the process of repair, meaning the appearance of fibrosis or bone neoformation (if present) and the presence of BMP7 in the addition mass and/or in the newly formed periodontal tissue. All the dento-osseous samples were fixed in formalin $10 \%$ for 48 hours, decalcified in trichloroacetic acid/hydrochloric acid for 1 week and longitudinally sectioned, perpendicularly to the bone addition area (Figures 1C).

$H E$ staining. From the treated group three pieces were collected, from which 21 dentoalveolar samples were sectioned. From the control untreated group, two samples were taken, from which 14 dentoalveolar samples were obtained. Then they were embedded in paraffin and cut at four microns, being subsequently stained with hematoxylin-eosin (HE).

Immunohistochemical staining. For logistical reasons, only 12 slides from the treated group and four slides from the untreated group were immunohistochemically stained and evaluated. Immunohistochemical staining for BMP7 expression was performed on 4 micron sections displayed on silanized slides. BMP7 antigens were unmasked using sodium citrate buffer, $\mathrm{pH}$ 6.0. The primary antibody used was rabbit polyclonal Anti-BMP7 antibody predicted to work with sheep (ab56023, Abcam, Cambridge, UK), diluted at 1:200. The detection kit "Super Sensitive TM Polymer-HRP IHC Detection System" (BioGenex, Hague, Netherlands) was used according to the protocol, with secondary HRP-conjugated anti-rabbit antibodies diluted in the blocking solution for an hour, subsequently revealed with the DAB chromogen (3,3'-diaminobenzidine). This caused a brown staining of antigen positive sites, against a blue background, obtained with hematoxylin counterstaining. On the negative control slides, primary antibodies were excluded.

Statistical analysis. A statistical study was performed by evaluating and comparing the two groups (treated and control). For this purpose, several parameters were quantified as markers of periodontal complex regeneration: the biomaterial and the cellular biomaterial (exclusively 

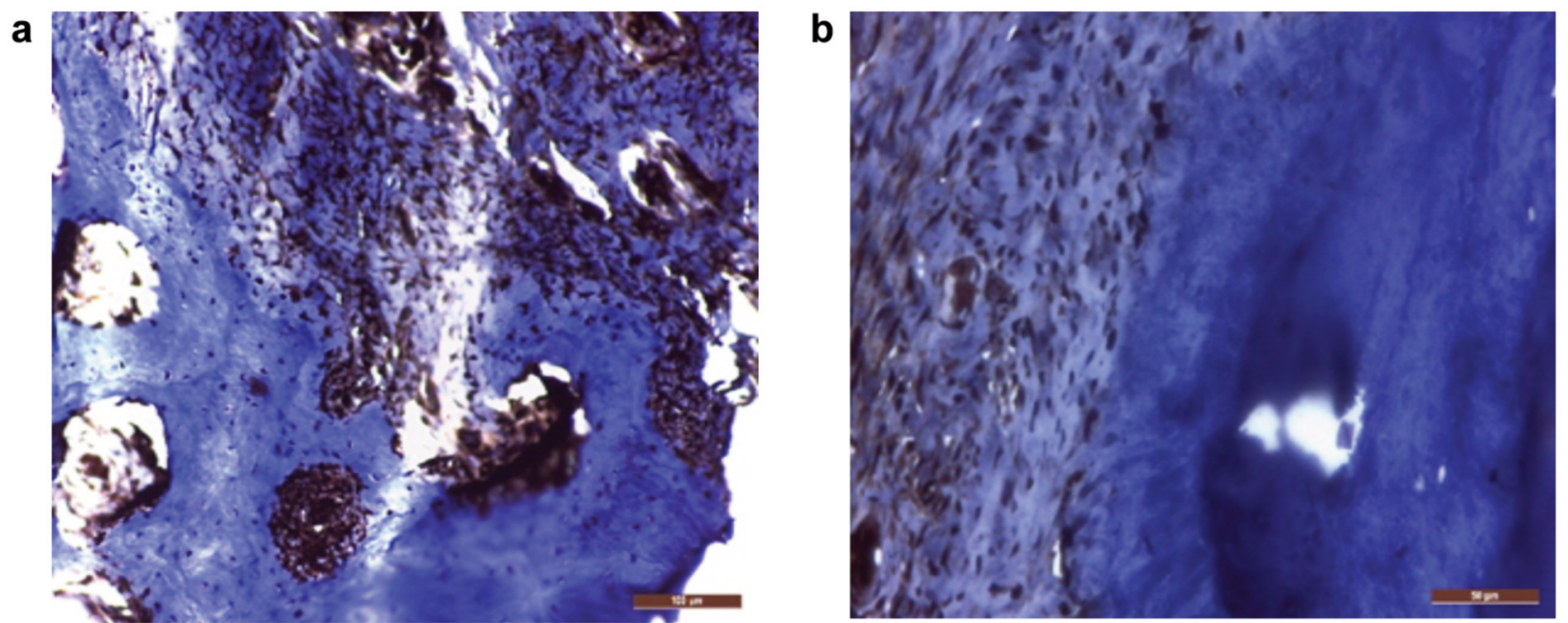

Figure 3. BMP7 expression in: (a) osteocytes and osteoblasts in the remaining original bone (200x) and (b) the desmodontal fibrocytes (400x). Immunohistochemical staining; positivity is indicated by the brown color of the cytoplasm and/or nuclei, on a blue background.

in the treated group), the fibroblasts/fibrosis, the newly formed cartilage and bone, osteocytes, osteoblasts, desmodontal ligaments, cellular cementum, cementum and mucosa (in both groups). These parameters were quantitatively evaluated in the HE stained histological preparations. The quantitative and qualitative BMP7 expression was also assessed. The bone addition score was determined by adding the grades of 12 parameters. To assess bone addition as well as biomaterial differentiation for each evaluated parameter, values from 0 to 3 ( $0=$ absent, $1=5 \%-25 \%, 2=25 \%-60 \%$, $3=>60 \%$ ) were used; these represented the amount of tissue found on the histological slides by direct visual assessment by two different examiners (any differences being next mediated by discussion). The total score represented the sum of the evaluated parameters. The highest possible value of the bone addition score was 36 points. The categories were established depending on the presence and extensions of: biomaterial, cellular biomaterial, cartilage and biomaterial, cartilage, new bone and biomaterial, new bone, osteocytes, osteoblasts, cellular cementum, cementum, desmodontal ligaments, mucosa. The analyzed elements depended on the characteristics of the differentiating periodontal tissues, similarly to previous studies (24). The statistical study was performed by evaluating and comparing the samples collected from the treated and untreated/control groups. First, the obtained data were presented as descriptive statistical parameters: median $\left(25^{\text {th }}-75^{\text {th }}\right.$ percentile). The statistical test used for comparison was the Mann-Whitney $U$-test. The relationship between the analyzed parameters was assessed by Pearson correlation in the case of data without outliers and with Spearman correlation in the case of data with outliers. A $p$-value less than 0.05 was considered statistically significant. Statistical analysis was performed using IBM Corp SPSS Version 25.0 and Microsoft Excel (2018) (Microsoft Corporation Armonk, New York).

\section{Results}

Regarding the control group, histological HE examination at 6 weeks after diagnosis revealed the persistence of chronic inflammatory infiltrate, periodontal desmodontal ligament and alveolar bone destruction, as well as presence of calculus deposits, bacterial plaque and foreign bodies in the desmodontal region, without reparatory signs (Figure 2A-D). Given the presence of chronic marginal periodontitis, BMP7 was evidenced by immunohistochemistry in the osteoblasts and osteocytes of the remaining initial bone (Figure 3A) and slightly in the desmodontium (Figure 3B), signaling an attempt to stimulate regeneration in response to the inflammatory bone destruction, while it was negative in all other sites.

In the treated group, at 6 weeks after the biomaterial mass addition, the histological evaluation on HE staining revealed important bone addition areas, represented by well-formed but thin new bone trabeculae growing on the vestibular part of the original alveolar bone, sometimes with islands of cartilage (cartilaginous ossification) (Figure 4D-J), adjacent to fibroblasts and desmodontal like fibrotic tissue (Figure 4C) and remnants of biomaterial with various degrees of cellularity (Figure 4A, B). In some areas we also observed new cellular cementum (Figure $4 \mathrm{~K}, \mathrm{~L}$ ). In this group, immunohistochemistry revealed the presence of BMP7 in all these areas, the highest expression (highest staining intensity) being observed in the biomaterial remnants (Figure $5 \mathrm{~A}, \mathrm{~B})$. However, the biomaterial showed a reduction of staining intensity with increasing cellularity, being less intense in the cellular biomaterial, containing undifferentiated mesenchymal cells (Figures 5A, B). The intensity of BMP7 expression was also gradually reduced in more differentiated areas, like those containing fibroblasts or newly formed cartilage and bone trabeculae (Figure 5C-F), however persisting in the periodontal desmodontium, lateral and also apical (Figure 5G, H) 

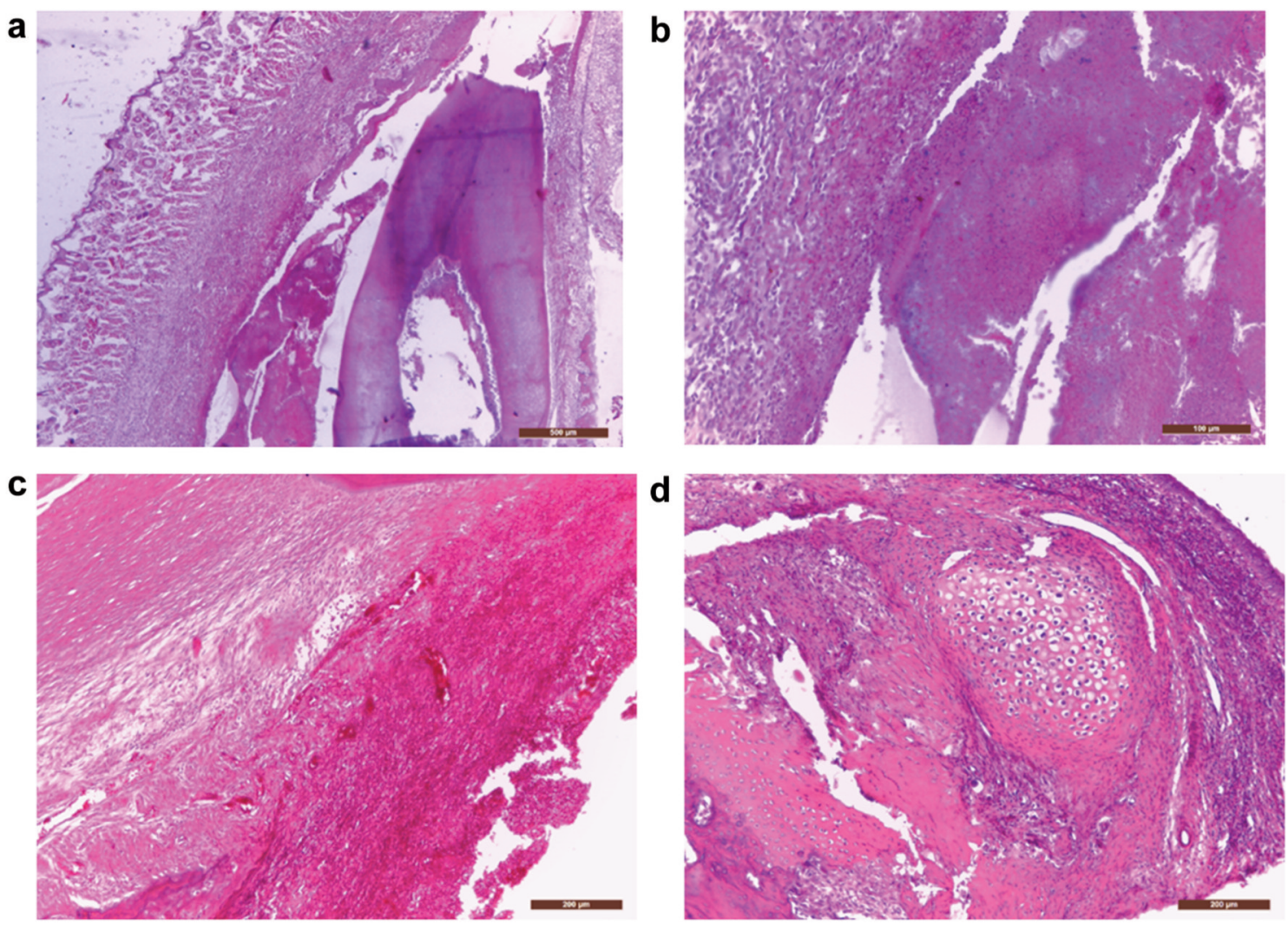

e
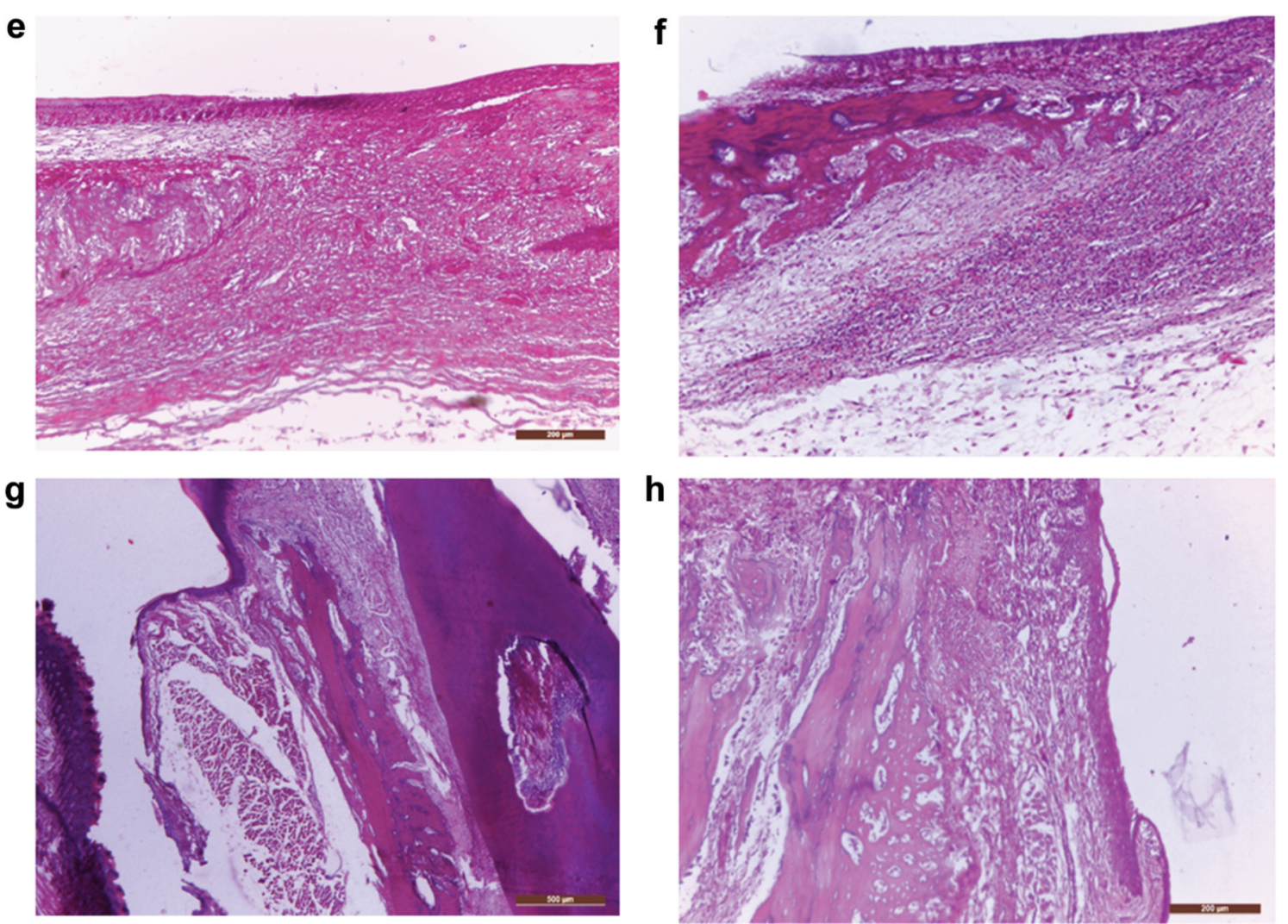

Figure 4. Continued. 

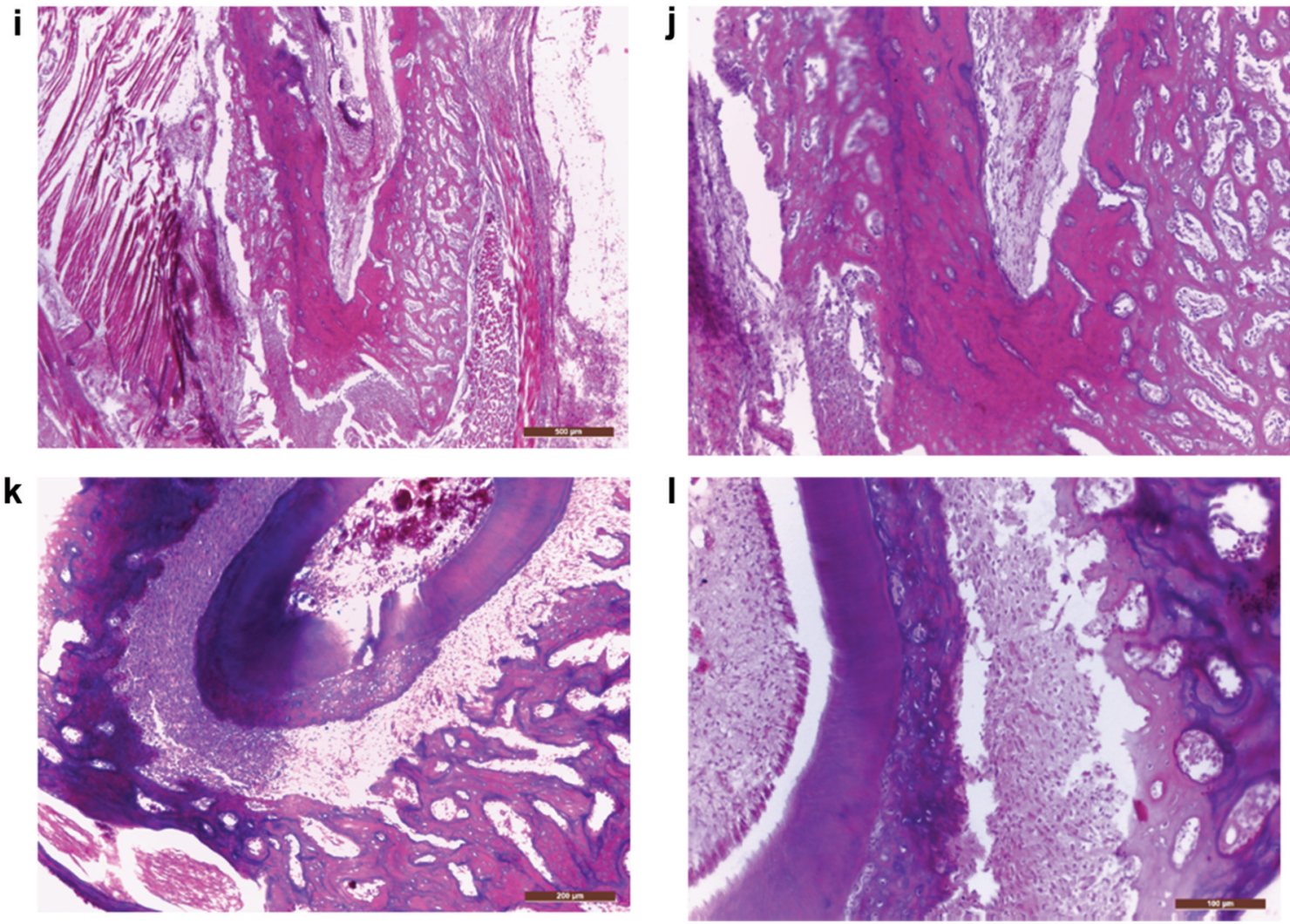

Figure 4. Biomaterial transformation during the regeneration of the periodontal complex (predominantly alveolo-desmodontal but also cemental): (a) Vestibular gingiva with biomaterial inoculated in a very deep sulcular pocket and the coronal portion of the root (40x); (b) Finely granular eosinophilic biomaterial and cellular biomaterial (200x); (c) Fibrous connective tissue, hypercellular fibroblastic area, hypercellular biomaterial and granular biomaterial (100x); (d) Old bone, new bone, cartilaginous island and hypercellular biomaterial (100x); (e) Cartilaginous regeneration of the alveolar bone, next to fibroblastic and hypercellular biomaterial (100x); (f) Old alveolar bone and attached to it, numerous delicate irregular trabeculae of new bone (100x); ( $g$ ) Lip, vestibular gingiva, new bone attached to the old alveolar bone, desmodontium and coronal portion of the root (40x); (h) Old alveolar bone, new bone and gingiva (100x); (i) Chin muscles, new bone, old bone, apical periodontal tissue (40x); (j) detail from (i) highlighting a longitudinal basophilic line which marks the limit between the new and the old bone (200x); ( $k$ ) old bone, desmodontium, apical part of the root, excessive cellular cementum (100x); (l) higher magnification of pulp, dentin, cellular cementum, desmodontium, old bone (200x). Hematoxylin-eosin staining.

Statistically, the parameters investigated on the untreated and treated groups were new bone formation, the presence of cartilage, fibroblasts and desmodontal like fibrotic tissue, the cellular and acellular biomaterial remnants, isolated or mixed with bone or cartilage, the new cellular cementum, the original destroyed cementum, the density of osteoblasts and osteocytes (for the control group from the original bone and for the treated group from the new bone) and separately, the BMP7 expression in each site.

In the treated group, multiple statistical correlations between the evaluated parameters were found, with the Spearman or Pearson $r$ correlation coefficient being statistically significant and positive, sometimes tending towards the maximum +1 , meaning the growth of one parameter accompanied closely the growth of the other one. Thus, the biomaterial was significantly correlated with: cellular biomaterial $(r=0.91, p<0.001)$, cartilage
( $r=0.52, p=0.005)$, cartilage+biomaterial $(r=0.68, p=0.008)$. Consequently, the amount of biomaterial led to a statistically significant increase in the amount of cellular biomaterial, and cartilage. The newly formed bone was significantly correlated with: new bone and biomaterial $(r=0.86, p<0.001)$, cellular cementum ( $r=0.71, p=0.003)$, cementum $(r=0.46, p=0.014)$ and osteocytes $(r=0.38, p=0.042)$. In addition, fibroblasts were significantly correlated with: newly formed bone $(r=0.70$, $p=0.001)$, new bone $(r=0.79, p<0.001)$, cementum $(r=0.76$, $p<0.001)$ and cellular cementum $(r=0.78, p<0.001)$.

Regarding BMP7, the amount of immunohistochemically positive biomaterial and desmodontal like fibrotic tissue was correlated with: new bone volume $(r=0.5, p=0.019)$, new bone and biomaterial $(r=0.75, p=0.072)$, cementum $(r=0.25$, $p=0.910)$ and cellular cementum $(r=0.75, p=0.315)$, providing a statistically significant correlation. 
a

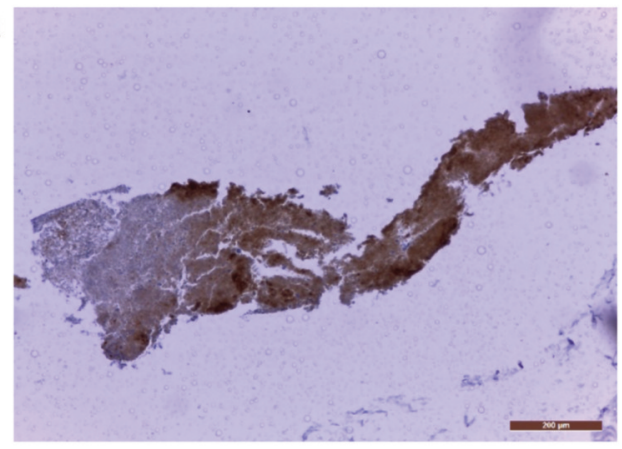

C

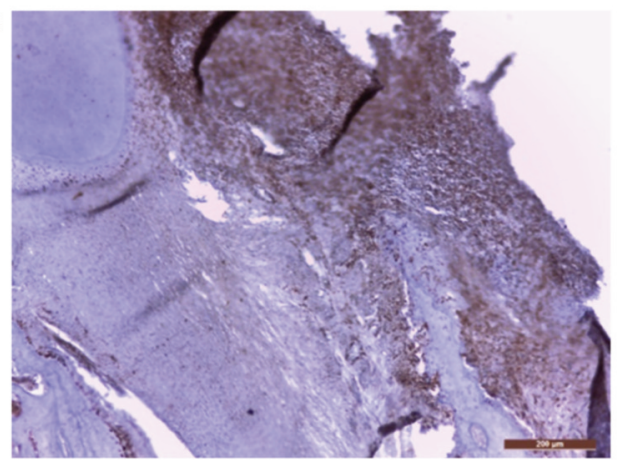

e

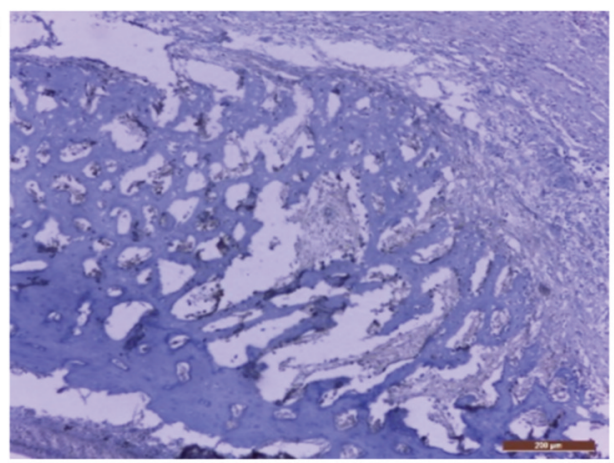

g

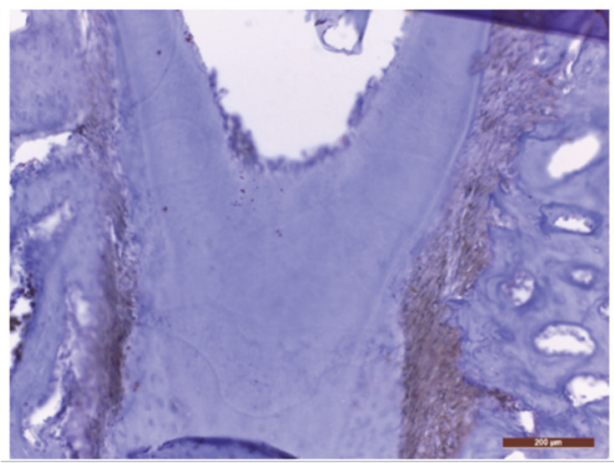

b

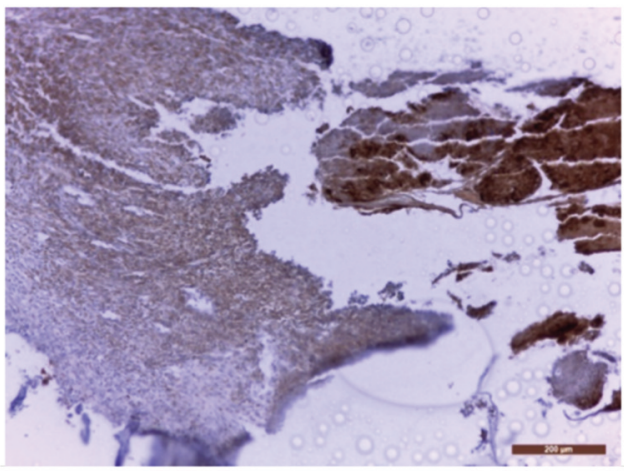

d

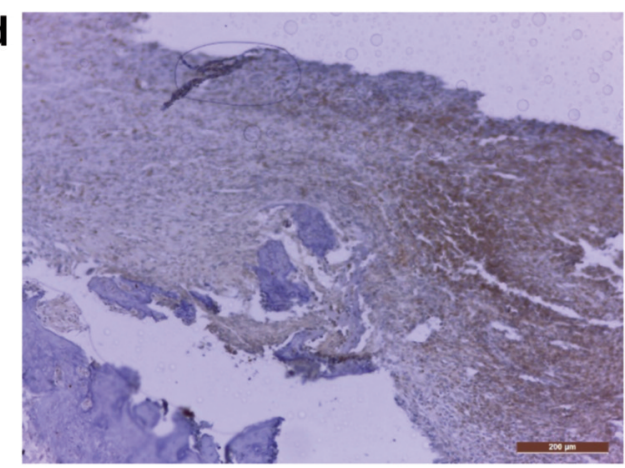

f

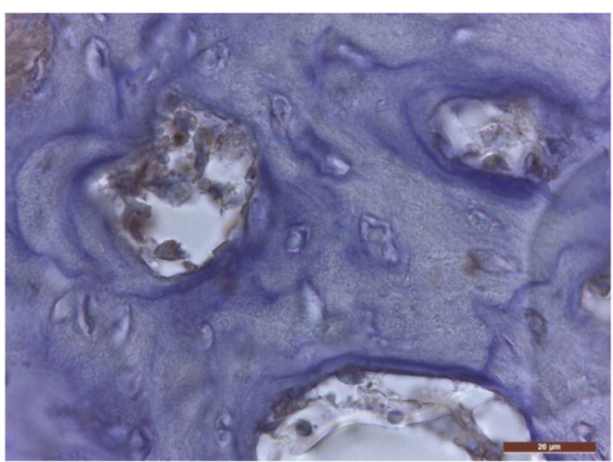

h

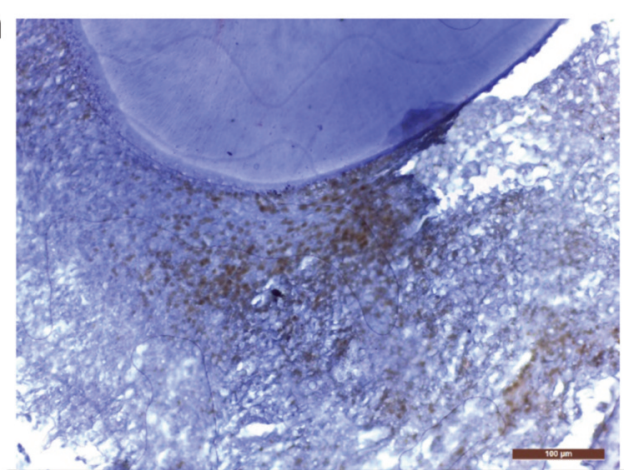

Figure 5. MBP7 expression in different areas of the periodontal complex from the treated teeth: (a) Positive biomaterial (40x); (b) Highly positive granular biomaterial and moderately positive cellular biomaterial (100x); (c) Transforming biomaterial, at the junction between the root and the alveolar old bone and desmodontal remnants; decreasing intensity from the cellular part to the fibrously transformed biomaterial (100x); (d) Moderately positive cellular biomaterial and less intense fibroblastic biomaterial (100x); (e) Delicate trabeculae of new bone, presenting slightly positive osteoblasts/osteocytes, and negative old bone (100x); (f) Positive osteoblasts and some osteocytes in new bone trabeculae, at very high magnification (1000x). (g) Moderately positive lateral periodontal desmodontium and negative cellular cementum, dentin and old alveolar bone (100x); (h) Moderately positive apical periodontal space and negative root (200x). Immunohistochemical staining; positivity is indicated by the brown staining of the cytoplasm and/or nuclei on a blue background. 
Table I. Comparison of the evaluated parameters (each graded from a minimum of 0 to a maximum of 3 ) and the bone addition total score between the control and treated groups.

\begin{tabular}{lccr}
\hline Parameters & $\begin{array}{c}\text { Control group }(\mathrm{n}=14) \\
\text { Median value } \\
\text { (minimum; maximum value) }\end{array}$ & $\begin{array}{c}\text { Treated group }(\mathrm{n}=26) \\
\text { Median value } \\
\text { (minimum; maximum value) }\end{array}$ & $p$-Value \\
\hline Biomaterial & $0(0 ; 0)$ & $2.5(1 ; 3)$ & $\mathbf{< 0 . 0 0 1}$ \\
Cellular biomaterial & $0(0 ; 0)$ & $3(2 ; 3)$ & $\mathbf{< . 0 0 1}$ \\
Fibroblasts/Desmodontal like & $0(0 ; 0)$ & $0(0 ; 3)$ & $\mathbf{0 . 0 2 3}$ \\
Cartilage & $0(0 ; 0)$ & $1(0 ; 1)$ & $\mathbf{0 . 0 1 4}$ \\
Cartilage and biomaterial & $0(0 ; 0)$ & $1(0 ; 1.5)$ & $\mathbf{0 . 0 0 9}$ \\
New bone & $0(0 ; 0)$ & $0(0 ; 2.5)$ & $\mathbf{0 . 0 1 9}$ \\
New bone and biomaterial & $0(0 ; 0)$ & $0(0 ; 1)$ & 0.072 \\
Cellular cementum & $0(0 ; 0)$ & $0(0 ; 1.5)$ & 0.315 \\
Cementum & $0(0 ; 0.5)$ & $3(2.5 ; 3)$ & 0.921 \\
Osteocytes & $2(1.5 ; 3)$ & $2.5(2 ; 3)$ & 0.166 \\
Osteoblasts & $1.5(1 ; 3)$ & $0(0 ; 0)$ & 0.145 \\
Mucosa & $0(0 ; 0)$ & $\mathbf{1 4}(\mathbf{1 2} ; \mathbf{1 8})$ & 0.443 \\
Total score & $\mathbf{5}(\mathbf{3 . 5} ; \mathbf{6})$ & & $\mathbf{< . 0 0 1}$ \\
\hline
\end{tabular}

Bold values indicate statistical significance.

As expected, there were significant differences regarding the reparatory signs between the two groups (treated and untreated). The development of the bone addition mass, i.e. the new periodontal tissue, was very significant in the treated group; the differences between the two groups showed high statistical significance for each parameter as well as for the total bone addition score $(p<0.001)$ (Table I).

\section{Discussion}

Bovine xenografts were introduced into clinical practice in 1954 (25) and since then processing technologies have been constantly improving. At present, commercially available xenografts are much safer.

The Hypro-Oss preparation was introduced into clinical practice in the early 2000's, obtained by the lyophilization technology, which allows preserving the natural, crystalline structure of the components. It contains hydroxyapatite $(70 \%$ of osteoconductive bovine hydroxyapatite) and atelocollagen (30\% collagen type I without telopeptide, which maintains the triple helicoidal structure of the matrix and contains the naturally osteoinductive elements TGF- $\beta$, BMPs and IGF). Hypro-Oss is taken from the bone structure of young animals, given that growth factors and BMPs are quantitatively reduced in old bovines (26).

The term PRP was introduced in the 1970s to describe autologous preparations and platelet enrichment in the plasma concentrate (27). PRP was introduced in the 1990s by top scientists such as Whitman and Marx (28). The main objective of PRP was the isolation of the greatest amount of platelet growth factors associated with their collection and reuse. The technique involved $\mathrm{CaCl}_{2}$ or bovine thrombin addition, also requiring a long preparation time (1). In the early 2000's, injectable PRP was developed, which was obtained by a single centrifugation (29).

A-PRF was developed by Choukroun in 2001 in France (30). A-PRF is a platelet concentrate with a high immunological value, because it incorporates all the elements involved in the immune response and the healing of soft and hard tissues in the form of a fibrin membrane, obtained from a $10 \mathrm{ml}$ blood sample (1).

Our study demonstrated the capacity of the composite graft to initiate, promote and maintain osteogenesis and odontogenesis in sheep. The graft, with a complex structure, contained lyophilized bovine bone enriched with atelocollagen type 1, combined with PRP (enriched with heparin) and APRF (with a membrane role). Histological examination showed its gradual replacement. The correlations of tissue differentiation were also statistically quantified. Graft biodynamics was characterized according to previous similar studies; the ideal addition mass was represented by cell differentiation and differentiated regeneration of tissues that progressively replace the graft (31), which also demonstrates the combined release of growth factors and BMPs (13). The presence of a multitude of BMPs in bone grafts is known and supported; for autologous blood derivatives, we refer to BMP2 in the first place (1). This experimental study supports the literature hypothesis according to which BMP2 and BMP7 induce osteogenic and odontogenic tissue differentiation without affecting each other (32), while being classified as strong osteoinductors (33). The presence of BMP7, which also plays the role of enhancing ossification and bone regeneration modeling as well as collagen accumulation (34) was demonstrated here by immunohistochemistry. 
BMP7 is generally expressed after two weeks during the healing process following trauma or interventions on bone structures (2). PRP rapidly releases proteins in high amounts (35), thus being considered an alternative to the use of rhBMP7 (36). A-PRF releases more growth factors compared to PRP in a period of up to ten days (35). In the case of the graft used, BMP7 was seen even after a period of six weeks due to sequential BMP release, favored by heparin and collagen, which facilitates cell proliferation and differentiation. Collagen, found in A-PRF and in the bovine xenograft, is considered a protein carrier (2), being at the same time an indicator of osteoblast differentiation (34). The test tubes utilized for the production of PRP contain heparin; heparin has the capacity to bind various growth factors (including TGF- $\beta$ ) (2) and at the same time, BMPs. The latter (being hydrophilic when released) are limited, having low bioavailability due to their poor stability and short biological half time in the circulatory system (31). The binding effect provided by heparin and collagen, respectively, was demonstrated in our study.

There is scientific evidence of resemblances of periodontitis development between sheep and humans (37, 38). Thus, BMP7 plays an important role in desmodontal development in both species, triggering the development of periodontal ligament (PDL) and cell differentiation into osteoblastic and cementoblastic phenotypes and, at the same time, playing a key role in the transformation of mesenchymal cells into bone and cartilage (39). In our study, BMP-7 was observed extensively in the experimental group in the desmodontal regeneration areas, and also in the control untreated group in the chronic marginal periodontitis areas. In the treated experimental group BMP-7 was locally synthesized, mediating the differentiation of cell lines such as cementoblasts and osteoblasts $(37,40)$ and also playing an anabolic and anticatabolic role (41).

Compared to other addition masses, e.g. rhBMP7 (released in the form of microspheres) (31), which starts to replace the biomaterial with bone structure only during the eighth week, our composite graft presents newly formed bone as early as the sixth week (confirmed by the presence of BMP7). In addition, this composite graft model does not produce catabolic effects $(13,41)$ or complications such as cell apoptosis, extensive inflammatory reactions (sometimes with vital risk), fibroblast infiltration, abnormal stimulation of bone resorption, ectopic ossification or increased oncogenic risk, as demonstrated in the case of rhBMP7. Furthermore, compared to bone autografts, additional surgeries with bone sacrifices were avoided.

Statistically, the comparison made between the two groups led to promising results. Histologically, the differences between the two groups (untreated and treated) were clear; however, due to the lack of newly formed cartilaginous elements and new bone in the untreated group and to the small number of samples, statistically significant differences were found only in the "new bone" and "biomaterial" categories. Compared to the untreated group, the treated group showed the highest significant statistical differences for the cell differentiation and biomaterial $(p<0.001)$.

\section{Conclusion}

The results of this study are compatible with those regarding the differentiation of osteo-periodontal tissues of the biomaterial studied in vivo. The current composite graft meets all the optimal conditions required for alveolar bone regeneration, due to the inclusion of the xenograft components (osteogenic cells, osteoconductive factors, porous osteoconductive matrix), as well as through the additional sequential release of growth factors and BMPs found in the autologous blood derivatives (including BMP7). Regarding autologous platelet plasma, the use of test tubes with heparin addition proves to be effective, due to the ability of heparin to bind growth factors and BMP7.

Although more extensive studies on much larger animal samples evaluated after a longer time period are required, given the clinico-histological aspect of the graft transformation and the lack of complications, the evolution of such bone addition appears to be favorable and more useful compared to other commercially available materials.

\section{Conflicts of Interest}

Bogdan-Cătălin Alexandru, Monica Popa, Liviu Oana, Cosmina Ioana Bondor, CarmenGeorgiu, Camelia-Sidonia Lazăr, Alina Simona Şovrea, Anne-Marie Constantin, and Gabriela Dogaru declare that they have no conflicts of interest regarding this study.

\section{Authors' Contributions}

Bogdan-Cătălin Alexandru: Conceptualization, Methodology, Investigation, Writing of original draft, Visualization. Monica Popa: Conceptualization, Methodology, Visualization, Supervision. Liviu Oana: Conceptualization, Visualization. Cosmina Ioana Bondor: Conceptualization, Formal and Statistical analysis. Carmen Georgiu: Investigation, Writing-original draft. Camelia-Sidonia Lazăr: Investigation, Writing-review \& editing. Alina Simona Şovrea: Visualization, Supervision. Anne-Marie Constantin: Methodology, Investigation, Writing-review \& editing, Visualization. Gabriela Dogaru: Writing-review \& editing, Visualization.

\section{References}

1 Miron JR and Choukroun J: Platelet Rich Fibrin: A 2nd generation platelet concentrate. In: Platelet Rich Fibrin in Regenerative Dentistry: Biological Background and Clinical Indications. Miron JR, Choukroun J (eds.). Hoboken, New Jersey, Wiley Blackwell, pp. 1-54, 2017. 
2 Jo J-Y, Jeong S-I, Shin Y-M, Kang S-S, Kim S-E, C-M Jeong and J-B Huh: Sequential delivery of BMP-2 and BMP-7 for bone regeneration using a heparinized collagen membrane. Int $\mathrm{J}$ Oral Maxillofac Surg 44(7): 921-928, 2015. PMID: 25769221. DOI: 10.1016/j.ijom.2015.02.015

3 Kim Y, Kang BJ, Kim WH, Yun HS and Kweon OK: Evaluation of Mesenchymal Stem Cell Sheets Overexpressing BMP-7 in Canine Critical-Sized Bone Defects. Int J Mol Sci 19(7): pii E20732018, 2018. PMID: 30018197. DOI: 10.3390/ ijms 19072073

4 Chen L, Lu X, Li S, Sun Q, Li W and Song D: Sustained delivery of BMP-2 and platelet-rich plasma-released growth factors contributes to osteogenesis of human adipose-derived stem cells. Orthopedics 35(9): e1402-e1409, 2012. PMID: 22955409. DOI: 10.3928/01477447-20120822-29

5 Liu HY, Wu AT, Tsai CY, Chou KR, Zeng R, Wang MF, Chang WC, Hwang SM, Su CH and Deng WP: The balance between adipogenesis and osteogenesis in bone regeneration by plateletrich plasma for age-related osteoporosis. Biomaterials 32(28): 6773-6780, 2011. PMID: 21700330. DOI: 10.1016/ j.biomaterials.2011.05.080

6 Hoggatt J: Hot ortho-biologic topics at AAOS 2011†: plateletrich plasma and related growth factors generate excitement. BioDrugs 25(3): 197-202, 2011. PMID: 21627344. DOI: 10.2165/11592710-000000000-00000

7 Schuckert KH, Jopp S and Osadnik M: The use of platelet rich plasma, bone morphogenetic protein-2 and different scaffolds in oral and maxillofacial surgery-literature review in comparison with own clinical experience. J Oral Maxillofac Res 2(1): e2, 2011. PMID: 24421984. DOI: 10.5037/jomr.2011.2102

$8 \mathrm{Hu}$ ZM, Peel SA, Ho SK, Sándor GK and Clokie CM: Comparison of platelet-rich plasma, bovine BMP, and rhBMP-4 on bone matrix protein expression in vitro. Growth Factors 27(5): 280-288, 2009. PMID: 19637071. DOI: 10.1080/ 08977190903137819

9 Gianakos A, Zambrana L, Savage-Elliott I, Lane JM and Kennedy JG: Platelet-rich plasma in the animal long-bone model: an analysis of basic science evidence. Orthopedics 38(12): e1079-e1090, 2015. PMID: 26652328. DOI: 10.3928/01477447-20151120-04

10 Tomoyasu A, Higashio K, Kanomata K, Goto M, Kodaira K, Serizawa H, Suda T, Nakamura A, Nojima J, Fukuda T and Katagiri T: Platelet-rich plasma stimulates osteoblastic differentiation in the presence of BMPs. Biochem Biophys Res Commun 361(1): 62-67, 2007. PMID: 17632078. DOI: 10.1016/j.bbrc.2007.06.142

11 Malhotra A, Pelletier M, Oliver R, Christou C and Walsh WR: Platelet-rich plasma and bone defect healing. Tissue Eng Part A 20(19-20): 2614-2633, 2014. PMID: 24666439. DOI: 10.1089/ten.TEA.2013.0737

12 Kalén A, Wahlström $\mathrm{O}$, Linder $\mathrm{CH}$ and Magnusson P: The content of bone morphogenetic proteins in platelets varies greatly between different platelet donors. Biochem Biophys Res Commun 375(2): 261-264, 2008. PMID: 18706392. DOI: 10.1016/j.bbrc.2008.08.014

13 Brigaud I, Agniel R, Leroy-Dudal J, Kellouche S, Ponche A, Bouceba T, Mihailescu N, Sopronyi M, Viguier E, Ristoscu C, Sima F, Mihailescu IN, Carreira ACO, Sogayar MC, Gallet O, Anselme K. Synergistic effects of BMP-2, BMP-6 or BMP-7 with human plasma fibronectin onto hydroxyapatite coatings: a comparative study. Acta Biomater 55: 481-492, 2017. PMID: 28434979. DOI: $10.1016 /$ j.actbio.2017.04.013

14 Masuki H, Okudera T, Watanebe T, Suzuki M, Nishiyama K, Okudera H, Nakata K, Uematsu K, Su C-Y, Tomoyuki Kawase T: Growth factor and pro-inflammatory cytokine contents in platelet-rich plasma (PRP), plasma rich in growth factors (PRGF), advanced platelet-rich fibrin (A-PRF), and concentrated growth factors (CGF), Int J Implant Dent 2(1): 19, 2016. PMID: 27747711. DOI: 10.1186/s40729-016-0052-4

15 Isobe K, Watanebe T, Kawabata H, Kitamura Y, Okudera T, Okudera H, Uematsu K, Okuda K, Nakata K, Tanaka T and Kawase T: Mechanical and degradation properties of advanced platelet-rich fibrin (A-PRF), concentrated growth factors (CGF), and platelet-poor plasma-derived fibrin (PPTF). Int J Implant Dent 3(1): 17, 2017. PMID: 28466249. DOI: 10.1186/s40729017-0081-7

16 Danesh-Meyer MJ, Pack AR and McMillan MD: A comparison of 2 polytetrafluoroethylene membranes in guided tissue regeneration in sheep. J Periodontal Res 32(1 Pt 1): 20-30, 1997. PMID: 9085239. DOI: 10.1111/j.1600-0765.1997.tb01378.x

17 Al Taii M, Broberg M, Cathro P and Richards L: Standardisation of sheep model for endodontic regeneration/revitalisation research. Arch Oral Biol 65: 87-94, 2016. PMID: 26871985. DOI: 10.1016/j.archoralbio.2016.01.008

18 Schliephake H, Knebel JW, Aufderheide M and Tauscher M: Use of cultivated osteoprogenitor cells to increase bone formation in segmental mandibular defects: an experimental pilot study in sheep. Int J Oral Maxillofacial Surg 30(6): 531-537, 2001. PMID: 11829236. DOI: 10.1054/ijom.2001.0164

19 Langhoff JD, Voelter K, Scharnweber D, Schnabelrauch M, Schlottig F, Hefti T, Kalchofner K, Nuss K and von Rechenberg B: Comparison of chemically and pharmaceutically modified titanium and zirconia implant surfaces in dentistry: a study in sheep. Int J Oral Maxillofacial Surg 37(12): 1125-1132, 2008. PMID: 18977118. DOI: 10.1016/j.ijom.2008.09.008

20 Galatos AD: Anesthesia and analgesia in sheep and goats. Vet Clin North Am Food Anim Pract 27(1): 47-59, 2011. PMID: 21215889. DOI: $10.1016 /$ j.cvfa.2010.10.007

21 Özkan F, Çakır-Özkan N, Eyibilen A, Yener T and Erkorkmaz Ü: Comparison of ketamine-diazepam with ketamine-xylazine anesthetic combinations in sheep spontaneously breathing and undergoing maxillofacial surgery. Bosn J Basic Med Sci 10(4): 297-302, 2010. PMID: 21108611. DOI: 10.17305/bjbms. 2010.2675

22 Delis G, Batzias G, Theodosiadou E, Kounenis G and Koutsoviti-Papadopoulou M: Influence of the injection site on the pharmacokinetics of amoxicillin after intramuscular administration of a conventional and a long-acting formulation in sheep. J Vet Pharmacol Ther 32(5): 457-464, 2009. PMID: 19754912. DOI: 10.1111/j.1365-2885.2009.01064.x

23 Delis GA, Koutsoviti-Papadopoulou M, Theodosiadou E, Kounenis $G$ and Batzias GC: Peripheral distribution of amoxicillin in sheep and influence of local inflammation. Vet $\mathbf{J}$ 185(3): 310-316, 2010. PMID: 19709911. DOI: 10.1016/j.tvjl. 2009.06.005

24 Lucaciu O, Baciut M, Baciut G, Gheban D, Bran S, Hedesiu M, Nicola $C$, Soritau $O$ and Gui D: Bone regeneration in craniofacial reconstruction with particulate grafts obtained through tissue engineering. Particulate Sci Techn 27(6): 497-518, 2009. DOI: $10.1080 / 02726350903328548$ 
$25 \mathrm{Nacu}$ V: Metode biologice stimulatoare a procesului reparator osos. Curierul Med 3(309): 37-45, 2009.

26 Linkhart TA, Mohan S and Baylink DJ: Growth factors for bone growth and repair: IGF, TGF beta and BMP. Bone 19(1 Suppl): 1S-12S, 1996. PMID: 8830994. DOI: 10.1016/s87563282(96)00138-x

27 Ramaswamy Reddy SH, Reddy R, Babu NC and Ashok GN: Stem-cell therapy and platelet-rich plasma in regenerative medicines: a review on pros and cons of the technologies. J Oral Maxillofac Pathol 22(3): 367-374, 2018. PMID: 30651682. DOI: 10.4103/jomfp.JOMFP_93_18

28 Whitman DH, Berry RL and Green DM: Platelet gel: an autologous alternative to fibrin glue with applications in oral and maxillofacial surgery, J Oral Maxillofac Surg 55(11): 1294-1299, 1997. PMID: 9371122. DOI: 10.1016/s0278-2391(97)90187-7

29 Alves R and Grimalt R: A review of platelet-rich plasma: history, biology, mechanism of action, and classification. Skin Appendage Disord 4(1): 18-24, 2018. PMID: 29457008. DOI: $10.1159 / 000477353$

30 Shah R, Triveni MG, Thomas R and Mehta DS: An update on the protocols and biologic actions of Platelet Rich Fibrin in dentistry. Eur J Prosthodont Restor Dent 25(2): 64-72, 2017. PMID: 28590091. DOI: 10.1922/EJPRD_01690Shah09

31 Li X, Yi W, Jin A, Duan Y and Min S: Effects of sequentially released BMP-2 and BMP-7 from PELA microcapsule-based scaffolds on the bone regeneration. Am J Transl Res 7(8): 14171428, 2015. PMID: 26396672.

32 Taşlı PN, Aydın S, Yalvaç ME and Sahin F: Bmp 2 and Bmp 7 induce odonto- and osteogenesis of human tooth germ stem cells. Appl Biochem Biotechnol 172(6): 3016-3025, 2014. PMID: 24477555. DOI: 10.1007/s12010-013-0706-0

33Morimoto T, Kaito T, Matsuo Y, Sugiura T, Kashii M, Makino T, Iwasaki $\mathrm{M}$ and Yoshikawa $\mathrm{H}$ : The bone morphogenetic protein$2 / 7$ heterodimer is a stronger inducer of bone regeneration than the individual homodimers in a rat spinal fusion model. Spine $\mathrm{J}$ 15(6): 1379-1390, 2015. PMID: 25733023. DOI: 10.1016/ j.spinee.2015.02.034

34 Xue Z, Niu LY, An G, Guo YS, Lv SC and Ren XP: Expression of recombinant BMP-7 gene increased ossification activity in the rabbit bone mesenchymal stem cells. Eur Rev Med Pharmacol Sci 19(16): 3056-3062, 2015. PMID: 26367729.

35 Kobayashi E, Flückiger L, Fujioka-Kobayashi M, Sawada K, Sculean A, Schaller B and Miron RJ: Comparative release of growth factors from PRP, PRF, and advanced-PRF. Clin Oral Invest 20(9): 2353-2360, 2016. PMID: 26809431. DOI: 10.1007/ s00784-016-1719-1
36 Hoellig M, Westhauser F, Kornienko K, Xiao K, Schmidmaier $\mathrm{G}$ and Moghaddam A: Mesenchymal stem cells from reaming material possess high osteogenic potential and react sensitively to bone morphogenetic protein 7. J Appl Biomater Funct Mater 15(1): e54-e62, 2017. PMID: 28009419. DOI: 10.5301/jabfm. 5000333

37 Silva NS, Silveira JAS, Lima DHS, Bomjardim HA, Brito MF, Borsanelli AC, Dutra IS and Barbosa JD: Epidemiological, clinical and pathological aspects of an outbreak of periodontitis in sheep. Pesq Vet Bras 36(11): 1075-1080, 2016. DOI: 10.1590/ s0100-736x2016001100003

38 Alexandru BC, Popa M, Oana L, Georgiu C, Tabaran F, Macri A, Purdoiu R, Istrate D, Dogaru G, Lazăr C, Bosca B, Peștean $\mathrm{C}$, Constantin AM and Șovrea A: Induction of chronic marginal periodontitis in an experimental sheep model: clinical, radiological and histological evaluation. Rom Biotechnol Lett 25(3): 1511-1525, 2020. DOI: 10.25083/rbl/25.3/1511.1525

39 Açil Y, Yang F, Gulses A, Ayna M, Wiltfang J and Gierloff M: Isolation, characterization and investigation of differentiation potential of human periodontal ligament cells and dental follicle progenitor cells and their response to BMP-7 in vitro. Odontology 104(2): 123-135, 2016. PMID: 25757659. DOI: 10.1007/s10266-015-0198-1

40 Oralová V, Chlastáková I, Radlanski RJ and Matalová E: Distribution of BMP6 in the alveolar bone during mouse mandibular molar eruption. Connect Tissue Res 55(5-6): 357366, 2014. PMID: 25084210. DOI: 10.3109/03008207. 2014.951441

41 Abula K, Muneta T, Miyatake K, Yamada J, Matsukura Y, Inoue M, Sekiya I, Graf D, Economides AN, Rosen V and Tsuji K: Elimination of BMP7 from the developing limb mesenchyme leads to articular cartilage degeneration and synovial inflammation with increased age. FEBS Lett 589(11): 12401248, 2015. PMID: 25889639. DOI: 10.1016/j.febslet. 2015.04.004

42 Epstein NE: Commentary on research of bone morphogenetic protein discussed in review article: Genetic advances in the regeneration of the intervertebral disc. Surg Neurol Int 4(Suppl 2): S106-S108, 2013. PMID: 23646271. DOI: 10.4103/21527806.109452 